\title{
„Als Differentialgeometer war ich fast ein Niemand."
}

\author{
Jean-Pierre Bourguignon
}

\begin{abstract}
Jean-Pierre Bourguignon erhielt auf der gemeinsamen Jahrestagung von ÖMG und DMV in Salzburg die Ehrenmitgliedschaft der DMV - insbesondere für seine Verdienste um die Partnerschaft der Mathematik in

Frankreich und Deutschland. Er war Präsident der Société Mathématique de France und der European

Mathematical Society und wirkte viele Jahre im wissenschaftlichen Beratungsgremium des

Forschungszentrums Oberwolfach. Seit 2014 ist er Präsident des European Research Council (ERC). Am Rande der Salzburger Tagung gab er den Mitteilungen ein Interview.
\end{abstract}

\begin{abstract}
Herr Bourguignon, finden Sie noch die Zeit für Mathematik?

Ich versuche es. Ich lese jeden Tag Mathematik, um meinen Kopf in Form zu halten. Und ich versuche, jedes Jahr mindestens zwei Vorträge zu halten - wirklich mathematische Vorträge. Aber natürlich nimmt das European Research Council den Großteil meines Tages in Anspruch, zusammen mit einigen anderen Evaluationen. Das ERC ist mein tägliches Brot.
\end{abstract}

Schaffen Sie es dennoch Ihre Forschung voranzutreiben?

Ich versuche es auch. Aber ich habe immer das Gefühl, ich sollte mehr tun. Ich habe zwei Artikel auf dem Schreibtisch liegen, die ich abschließen möchte, und ein Buch, das ich zu Ende bringen will. Um etwas Mathematisches zu schreiben, brauche ich mindestens einen halben Tag; wenn ich nur eine Stunde habe, kann ich etwas lesen, aber um wirklich etwas zu schaffen, brauche ich eine längere Zeitspanne.

Ihre eigene Forschung liegt in der Differentialgeometrie.

Ja, ich wurde als Differentialgeometer ausgebildet. Mein Doktorvater war Marcel Berger. Mein Forschungsfeld war Differential- und insbesondere Riemannsche Geometrie. Aber ziemlich früh habe ich mich mit Fragen am Rande der theoretischen Physik beschäftigt, mit Eichtheorien, allgemeiner Relativitätstheorie und Spinoren. Ich habe das immer als Mathematiker betrieben. Dennoch war es zum Beispiel für die Eichtheorie sehr wichtig, dass ich als Student an der École Polytechnique in Paris ernsthaft Quantenmechanik gehört habe.

War eine solche Interdisziplinarität zwischen Mathematik und Physik damals üblich?

Nein, es war damals in Frankreich nicht üblich, als Mathematiker Quantenmechanik zu studieren. Mathematiker und Physiker waren klar getrennt. Als Mathematiker an der École Normale zum Beispiel machte man fast keine Physik. Zu dieser Zeit war es so: Wenn man nicht algebraische Geometrie oder Zahlentheorie betrieb, war man kein echter Mathematiker. Als Differentialgeometer war ich fast ein Niemand.

Sie sind trotzdem dabei geblieben.

Am Anfang war ich mir nicht sicher, ob für mich die Differentialgeometrie das Richtige ist. Das hat sich erst endgültig geändert, als ich die USA besuchte. Während meiner Zeit in Stanford bekam ich einen Anruf von Professor Chern Shiing-Shen. Er lud mich zum Mittagessen ein und wollte wissen, woran ich arbeite. Ich war völlig überrascht. Ich hatte noch nicht meine „Thèse d'État“ verteidigt. Ich nehme an, Marcel Berger hatte ihm gesagt, dass ich in Stanford bin. Später konnte ich erfahren, dass Professor Chern viele junge Leute nach ihrer Forschung fragte. Haben Sie Professor Chern mal getroffen?

Nein.

Nein, Sie sind zu jung. Er hatte eine großartige Art zuzuhören. Er sprach nicht viel, aber seine Fähigkeit zuzuhören war phantastisch. Professor Chern war immer an Neuem interessiert. Jedenfalls dachte ich nach diesem Mittagessen: Vielleicht ist es doch nicht so dumm, was ich mache.

Wie sind Sie zum ersten Mal in die USA gekommen?

Das kam durch Jim Simons und es ging sehr schnell - wie fast alles was er macht. Im Juni 1972 kam er nach Paris und hörte einen Vortrag von mir. Am nächsten Morgen erhielt ich ein Angebot für eine Stelle an der State University of New York at Stony Brook. Jetzt mussten wir uns schnell entscheiden, ob wir gehen oder nicht. Ich sage wir, weil ich damals schon verheiratet war und wir ein Kind hatten. Wir sind nach Amerika gezogen, aber ich bin nur knapp ein Jahr in Stony Brook geblieben. Dennoch war es eine sehr wichtige Zeit für mich. In jenem Jahr, 1972-1973, gab es in Stony Brook 14 Differentialgeometer in der Faculty. Das war für mich großartig. Als ich nach Frankreich zurückkam, hatte ich Kontakt zu den besten Differentialgeometern der Welt.

Wie entwickelte sich in den $1970 e r$ Jahren die Zusammenarbeit mit der theoretischen Physik? 


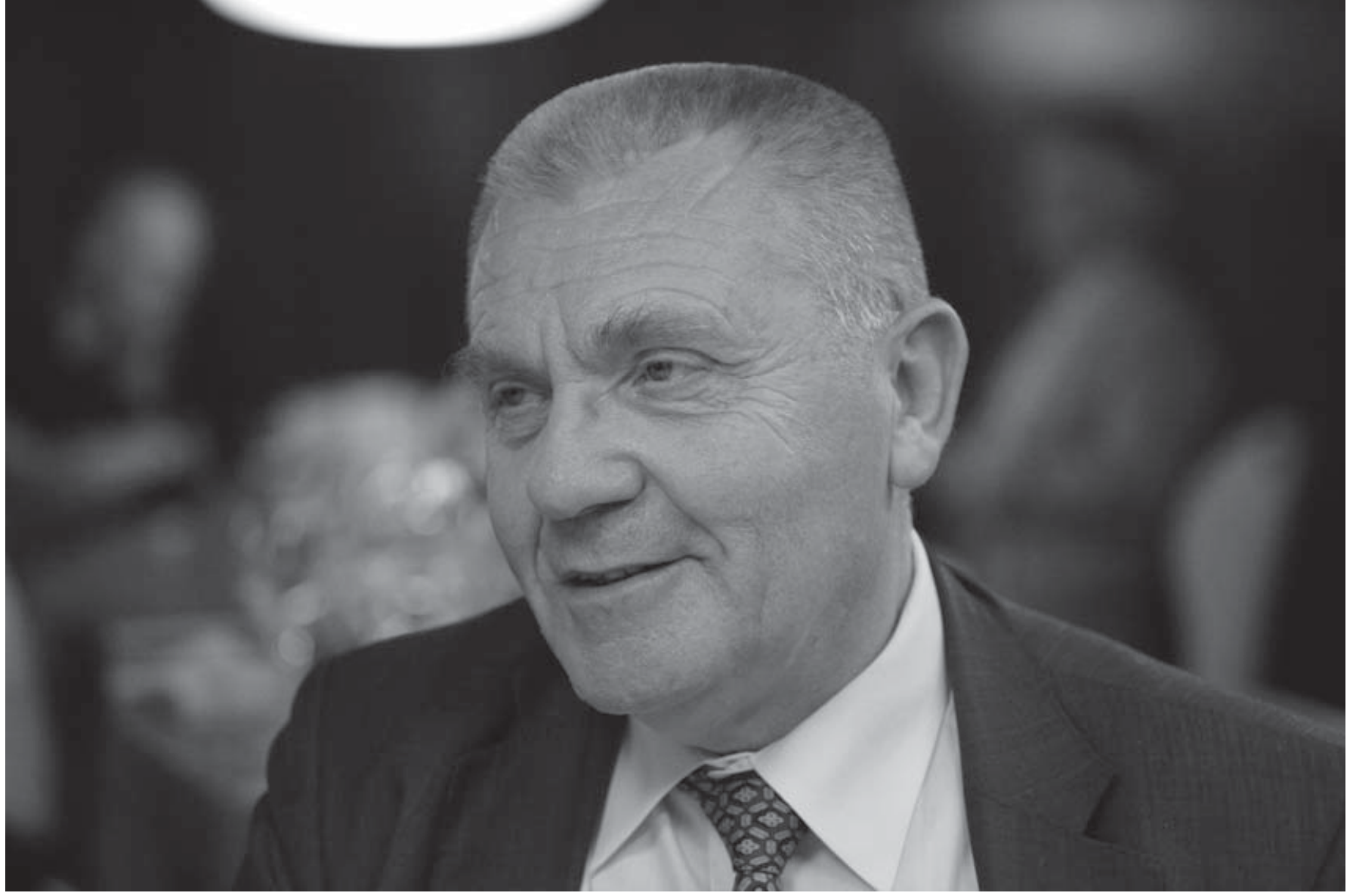

Jean-Pierre Bourguignon auf der Versammlung der Oberwolfach-Kommissionen 2012 (Foto: Gerd Fischer/Bildarchiv des Mathematischen Forschungsinstituts Oberwolfach)

Anfang der 7oer Jahre gab es für die Zusammenarbeit zwischen Differentialgeometern und theoretischen Physikern in der Eichtheorie zu wenige Mathematiker, die genug von Physik verstanden, um die Fragen richtig zu fassen. Die Fragen waren zwar rein mathematisch formuliert, aber um eine Frage richtig zu behandeln, ist es immer besser, wenn man weiß, weshalb sie für das andere Fach wichtig ist.

Sie waren einer derjenigen, die den Dialog mit der Physik gesucht haben.

Ja, aber die Anfänge der Diskussion mit den theoretischen Physikern waren schwierig. Beim ersten Versuch in Stony Brook ging es vollkommen schief. Jim Simons und Yang Cheng-Ning veranstalteten ein gemeinsames Seminar. Danach hatten wir das Gefühl, was sie machten, war für uns völlig uninteressant, und sie hatten das Gefühl, was wir machten, war irrelevant für sie. Es war noch zu früh. Erst in der zweiten Hälfte der 7 oer Jahre funktionierte es. Dann kamen die Differentialgeometrie und insbesondere das Thema Krümmung stark in Mode. Schließlich gab es im Jahr 1980 ein Sonderprogramm des IAS in Princeton zur globalen Analysis, an dem ich auch teilnahm. Dort kamen sehr viele großartige Experten zusammen. Es war ein ganz entscheidender Moment für das Gebiet und für meine eigene Arbeit.

Sie sind durch Jim Simons an die Stony Brook University gekommen und Sie haben später auch mit ihm und

\section{Blaine Lawson gemeinsam publiziert. Wie kam das?}

Das war eigentlich ein großer Zufall. Blaine und ich kannten uns schon länger gut. Er besuchte damals, 1979, IHÉS in Bures-sur-Yvette und ich wurde in dem Jahr von Physikern gebeten, eine Vorlesung über die differentialgeometrische Basis für Eichtheorie zu halten. In der Vorbereitung für die Vorlesung habe ich mit Blaine gesprochen. Ich habe ihm erklärt, was ich in der Vorlesung vorhabe. Dabei kam ich beiläufig auf eine Frage zu sprechen, die in der Physik schon länger offen war und an der ich arbeitete. Ich hatte etwa die Hälfte der Antwort auf diese Frage. Als ich das erwähnte, stellte sich heraus, dass er genau die andere Hälfte hatte. Wir hatten damals regelmäßig miteinander diskutiert, aber ich habe ihm vorher nie von diesem Halbresultat erzählt. Das kam erst durch die Vorlesung für die Physiker. Es zeigt, dass man oft nicht genug oder nicht offen genug mit seinen Freunden spricht.

\section{Das war die Arbeit „Stability and isolation phenomena of Yang-Mills fields"?}

Richtig. Die Idee kam ursprünglich von Jim Simons. Er hatte den Satz für Dimension 5 bewiesen. Für Physiker ist Dimension 4 die kritische Dimension und die Frage ist dann subtiler. Deshalb haben wir Jim Simons gefragt, ob wir die Ankündigung in den Proceedings of the US National Academy of Science gemeinsam machen wollen [Anm. d. Red.: „Stability and gap phenomena for YangMills fields"] Er hat zugestimmt und es wurde seine letzte 
Arbeit, bevor er in die Finanzwirtschaft wechselte. Wir haben natürlich nicht gewusst, dass er später Milliardär würde.

Die Zeit in den USA war sehr wichtig, sagen Sie. Aber Sie haben auch eine intensive Beziehung zu Deutschland gepflegt.

Ich habe mit sehr vielen deutschen Kollegen über viele Jahre eng zusammengearbeitet. Und das fand natürlich oft in Deutschland statt. Regelmäßige Einladungen von Professor Wilhelm Klingenberg nach Bonn waren dafür kritisch. Es gab jährlich die Arbeitstagung in Bonn, aber auch eine kleinere, auf Differentialgeometrie fokusiert. Es gab auch die Geometrietagungen in Oberwolfach, die für alle europäischen Differentialgeometeriker enorm wichtig waren. Ich selbst verbrachte das Jahr 1976/1977 in Bonn als Gastprofessor am SFB 40. Das Max-Planck-Institut gab es damals noch nicht in Bonn. Ich habe auch dort gelehrt. Es erlaubte mir, eine besondere Beziehung zu Professor Friedrich Hirzebruch zu entwickeln. Was die deutsche Sprache betrifft, hatte ich in der Schule zehn Jahre lang Deutsch gelernt und später viel deutsche Literatur gelesen. Aber Deutsch zu sprechen fiel mir damals doch schwer.

Das Bonner Max-Planck-Institut gab es noch nicht, als Sie das erste Mal in Bonn waren. Aber für das Leipziger Max-Planck-Institut für Mathematik in den Naturwissenschaften waren sie so etwas wie ein Geburtshelfer.

Was Sie sagen ist sicher übertrieben. Das war eine sehr interessante Erfahrung. Sie wissen sicher, dass das Bonner Max-Planck-Institut erst im fünften Anlauf zustande kam. Und nun sollte es - auch auf Initiative von Professor Hirzebruch und mit der Unterstützung von Jürgen Moser und Stefan Hildebrandt - ein weiteres Max-PlanckInstitut für Mathematik geben. Andere Wissenschaftler der Max-Planck-Gesellschaft sagten: Das haben wir einmal gemacht, das machen wir auf keinen Fall wieder. Und wenn wir es machen, dann organisieren wir es so, wie wir als MaxPlanck-Wissenschaftler uns das vorstellen, und nicht wie die Mathematiker sich das denken. Es hat einige Mühe gekostet, es so einzurichten, wie es für Mathematiker sinnvoll ist. $\mathrm{Zu}$ Anfang des Instituts war ich ein Mitglied des wissenschaftlichen Beirats für das Leipziger Max-Planck-Institut.

Ein Europäisches Projekt für das Sie mit vielen deutschen Mathematikern zusammengearbeitet haben, war das Zentralblatt MATH, heute zbMATH. War das ähnlich schwierig?

Die ursprüngliche Idee war eine Zusammenarbeit mit MathSciNet zu schaffen. MathSciNet und das Zentralblatt haben, wie Sie sicher wissen, einen gemeinsamen Gründer, nämlich Otto Neugebauer. Er hatte 1931 das Zentralblatt für Mathematik und ihre Grenzgebiete gegründet. Später musste er vor den Nazis in die USA fliehen. Dort hat er gewissermaßen ein anderes Zentralblatt gegründet. Das wurde MathSciNet.

Es erschien uns als Europäern logisch, beide zusammenzuführen. Die Antwort von MathSciNet war: Kein
Problem! Gebt uns die Daten, wir machen alles, ihr bekommt freie Kopien. Das war natürlich nicht, was wir wollten. Also haben wir Springer als Verlag, das FIZ in Karlsruhe [heute Leibniz Institut für Informationsinfrastruktur, Anm. d. Red.], die Heidelberger Akademie der Wissenschaften und die Europäische Mathematische Gesellschaft in ein Boot geholt, um ein europäisches, ursprünglich deutsches System aufzubauen. Bernd Wegner spielte eine kritische Rolle für die Weiterentwicklung der Datenbasis. Es gab auch viele Beiträge aus Frankreich. Ein Teil der Software wurde zum Beispiel in Grenoble erstellt.

Wenn Sie beide Systeme heute vergleichen, würden Sie sagen, es war die Mühe wert?

Die beiden sind sehr ähnlich, wenn auch nicht ganz gleich. Die Qualität der Daten ist sehr vergleichbar. Es ist sicher, dass der Wettbewerb beide Systeme positiv beeinflusst hat. Einen großen Unterschied gibt es jedoch: MathSciNet macht mehr Geld, da der geschlossene Markt für MathSciNet viel größer ist, für zbMath die Kosten aber ungefähr dieselben sind. Dennoch finde ich es richtig und wichtig, dass man für das Zentralblatt als europäisches Projekt gekämpft hat.

Wir könnten sicher noch lange darüber sprechen, was Sie auf institutioneller Ebene für die Mathematik in Deutschland getan haben. Sie waren zum Beispiel Editor für die Mathematischen Annalen, Sie waren im Beirat für Oberwolfach. Aber Sie haben auch enge persönliche Beziehungen zur deutschen Mathematik.

Ich erinnere mich an einen Besuch in Hamburg auf Einladung von Professor Oswald Riemenschneider. Ich hielt einen Vortrag über Eichtheorie und es gab einen Zuhörer, der sehr viele, tiefe Fragen stellte. Nach dem Vortrag fragte ich Professor Riemenschneider, wer das sei. Es war Erich Kähler. Damals war für mich Kähler jemand wie Élie Cartan. Aber er war viel jünger als ich gedacht hatte. Natürlich war er damals schon etwa 80 , aber noch extrem energetisch und scharf. Ich kannte selbstverständlich seine Arbeiten. Der Artikel, in dem er Kählersche Geometrie einführte, ist hervorragend: jede halbe Seite enthält eine neue Idee und jede Idee ist entscheidend. Aber ich hatte nie überprüft, wann das entstanden ist. Er war tatsächlich sehr jung, als er ihn schrieb. Wir haben dann noch den ganzen Abend miteinander diskutiert.

Beeinflussen Sie die Erfahrungen Ihrer eigenen wissenschaftlichen Laufbahn, wenn Sie heute als Präsident des ERC arbeiten?

Man muss zunächst einmal folgendes sehen: Als ich Beamter am CNRS wurde, war ich nur 21. Damals war das Leben für junge Wissenschaftler viel leichter. Heute müssen selbst die besten sehr lange warten, bis sie endlich eine feste Stelle bekommen. Viele Stellen sind sehr kurz bemessen, ein Jahr, zwei Jahre oder vielleicht drei. Deshalb ist es sehr wichtig für den ERC, dass durch dieses Programm Wissenschaftlerinnen und Wissenschaftler für fünf Jahre gefördert werden. In fünf Jahren kann man etwas Ernsthaftes leisten. 
Sie sagen, Sie hatten es damals leichter?

Ja, wenn man meine Zeit mit heute vergleicht, dann war es für uns freundlicher. Ich war mit 23 Vater. Das war möglich, weil ich eine Stelle hatte und meine Frau ebenfalls. Im CNRS hatte ich auch die Möglichkeit, ein Jahr in den USA zu verbringen. Ich musste nur entscheiden, ob ich mein Gehalt in Frankreich behalten oder in den USA ein Gehalt in Dollar beziehen wollte. Die Währungsschwankungen waren damals empfindlich. Leider habe ich mich dabei jedesmal falsch entschieden! Mit einer jungen Familie ins Ausland zu gehen, barg natürlich auch damals Risiken.

In Ihrem heutigen Amt haben Sie viele Aufgaben in der politischen Sphäre. Ist es von der intellektuellen Ausstattung her eher von Vorteil oder von Nachteil, Mathematiker zu sein?

Das ist vielleicht etwas, was Mathematiker schnell falsch einschätzen. Ihr Fach wird von vielen Menschen sehr positiv gesehen. Die Leute wissen, dass es nahezu unmöglich ist, in der Mathematik Karriere zu machen, ohne wirklich etwas zu leisten. Hinzu kommt, dass Mathematiker nicht so viel Geld brauchen. Deshalb machen sich
Kollegen von anderen Disziplinen weniger Sorgen, dass ein Mathematiker alles Geld auf die Seite der Mathematik schaffen will. Auch ist im Durchschnitt der Wettbewerb in der Mathematik vielleicht nicht so hart wie in anderen Disziplinen. Von daher ist es ziemlich gut, Mathematiker zu sein. Die andere Seite der Geschichte ist, dass Mathematiker oft für verschlossen gehalten werden. Für mich war das kein Problem. Ich habe immer die Kooperation mit anderen Wissenschaftlerinnen und Wissenschaftlern gesucht, insbesondere mit Physikern am IHÉS. Ich hatte auch viele Kontakte in der Industrie, weil es mich interessiert hat, mit Ingenieuren zu sprechen. Und ich habe oft den Austausch mit Philosophen und Künstler gesucht. Als junger Mann dachte ich, ich würde Philosoph. Im Ganzen ist die Außenwahrnehmung der Mathematiker gut: wir gelten als Leute, die sich für die Wissenschaft echt interessieren. Und wenn man dann noch zeigt, dass man bereit ist, mit anderen zu sprechen und zuzuhören, dann ist man als Mathematiker, denke ich, sehr geschätzt.

Mit Jean-Pierre Bourguignon sprach Sebastian Stiller.

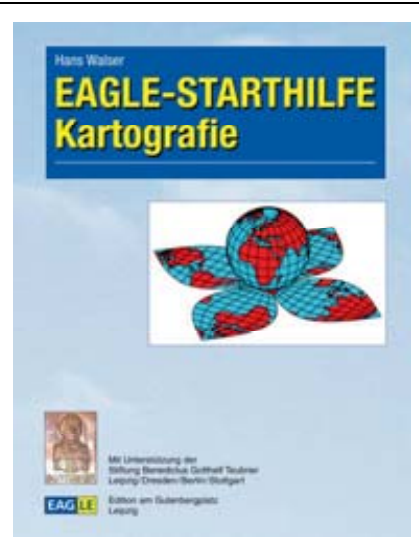

H. Walser. EAGLE 098. 1. A. 2017. 978-3-95922-098-9

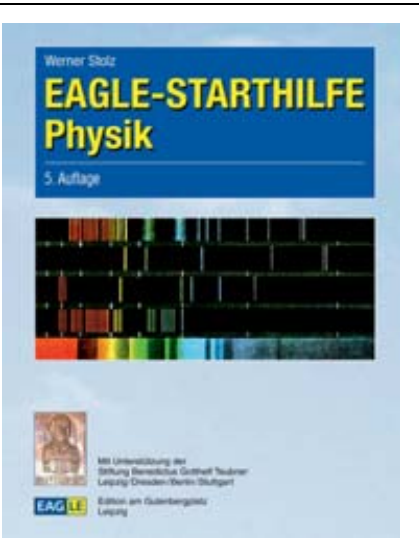

W. Stolz. EAGLE 086. 5. A. 2015. 978-3-95922-086-6

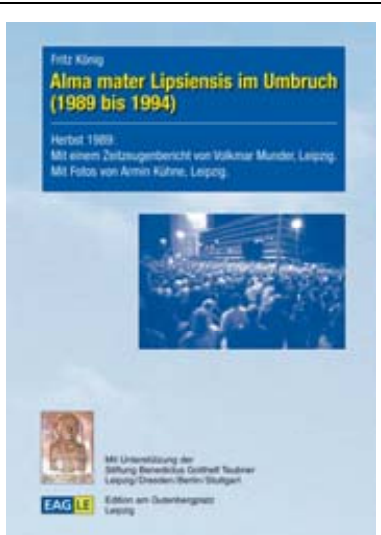

F. König. EAGLE 099.

1. A. 2017. HC. 978-3-95922-099-6

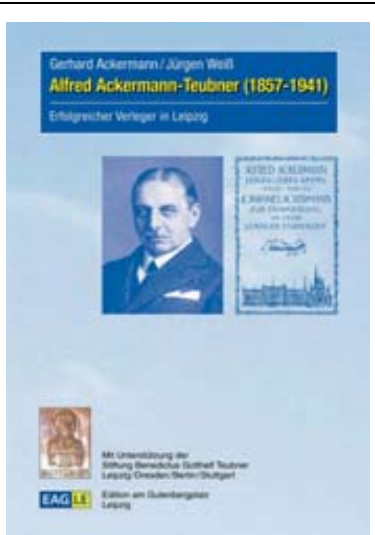

G. Ackermann / J. Weiß. EAGLE 090. 1. A. 2016. HC. 978-3-95922-090-3

\section{EAG.LE}

Edition am Gutenbergplatz Leipzig: www.eagle-leipzig.de I www.eagle-leipzig.de/starthilfen.htm I https://twitter.com/EagleLeipzig NEU/September 2017: Hans Walser, EAGLE-STARTHILFE Kartografie. EAGLE 098. Von 1995 bis 2003 erschienen 17 Teubner-Starthilfen. Nach dem Verkauf von Teubner an Bertelsmann waren keine Starthilfen mehr möglich. Seit 2004 erscheinen in Leipzig EAGLE-STARTHILFEN.

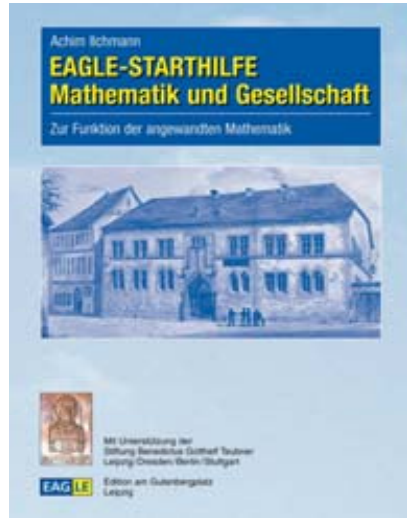

A. Ilchmann. EAGLE 093 1. A. 2016. 978-3-95922-093-4

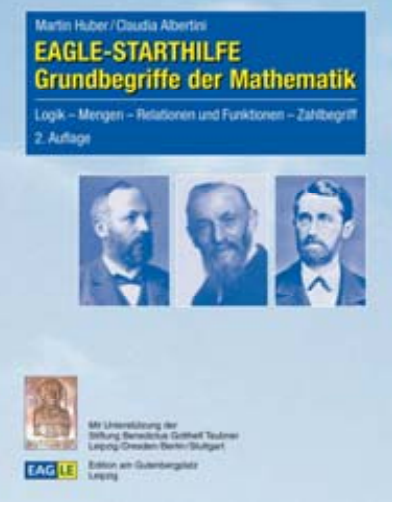

M. Huber / C. Albertini. EAGLE 072. 2. A. 2015. 978-3-937219-97-4

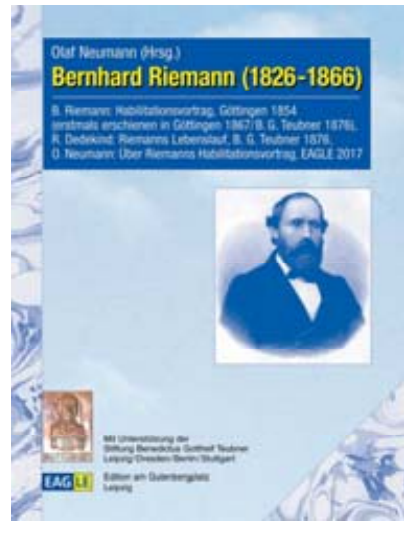

O. Neumann (Hrsg.). EAGLE 097. 1. A. 2017. HC. 978-3-95922-097-2

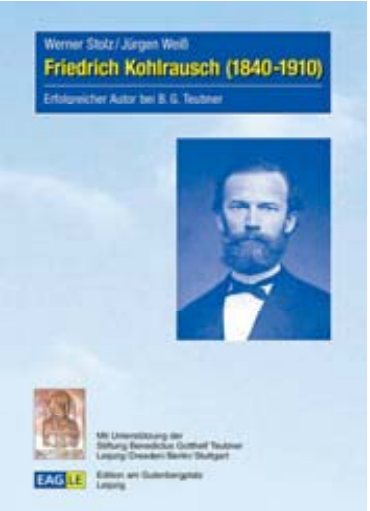

W. Stolz / J. Weiß. EAGLE 095. 1. A. 2017. HC. 978-3-95922-095-8 